\title{
Study of $v_{\mu}$-CC Interactions in the Resonance Region Using Nomad Data
}

\author{
Hongyue Duyang* \\ University of South Carolina \\ E-mail: duyangeemail.sc.edu
}

\begin{abstract}
Neutrino induced pion production in the resonance region is one of the most important interaction modes for the current and future generation long-baseline neutrino oscillation experiments. It is also sensitive to nuclear effects, including fermi motion, initial-state nucleon correlations, and final-state interactions, etc., which affect event topology and energy reconstruction of resonance interactions, and contribute to the systematic uncertainty for oscillation measurements. We present a study of the $v_{\mu}$ charge current interactions in the resonance region using high-statistics, high-resolution NOMAD data. Constraint on nuclear effects is also discussed.
\end{abstract}

The 21st international workshop on neutrinos from accelerators (NuFact2019)

August 26 - August 31, 2019

Daegu, Korea

${ }^{*}$ Speaker. 
Neutrinos can inelastically scatter off target nucleons via charged current (CC) interactions. In hadronic invariant mass $W<2.0 \mathrm{GeV}$ region, resonance interactions dominate in which a resonant state $\left(\Delta, N^{*}\right)$ of the excited target nucleon is created and decay immediately into a nucleon and a single pion.

Resonance is the dominant interaction channel for the next generation long-baseline neutrino experiments in the few-GeV energy region. Moreover, resonance interactions are sensitive to nuclear effects, including nucleon-nucleon correlations, Fermi motion, and final-state interactions (FSI), etc., which often produce neutrons or low-energy charged particles difficult to detect or measure precisely in neutrino detectors. Since the oscillation probability is measured as a function of neutrino energy, it is important for neutrino oscillation experiments to understand nuclear effects in resonance interactions for correct neutrino energy reconstruction. The resonance interactions are commonly simulated by Rein-Seghal (RS) model [1] in neutrino generators such as GENIE [2].

The Neutrino Oscillation MAgnetic Detector (NOMAD, WA-96) [3] is designed to search for $v_{\mu}$ to $v_{\tau}$ oscillation in the CERN SPS wide band neutrino beam $(2.5 \mathrm{GeV}$ to $300 \mathrm{GeV})$ [4][5]. The NOMAD detector is a 2.7 ton detector consisting of Drift Chambers, Transition Radiation Detector, Preshower Detector, Calorimeter, and Muon Chambers, etc. (Fig 1, left). The detector is placed in a $0.4 \mathrm{~T}$ dipole magnetic field for the identification of particle charges and precise measurement of particle momentums. The full NOMAD dataset with about 4 million recorded neutrino interactions is used in this measurement for statistical precision. GENIE neutrino generator (v2.10.2) is used to simulate neutrino interaction in the NOMAD detector. No additional tuning is applied to the GENIE simulation before being compared to the NOMAD data. The detector response is simulated by a package based upon GEANT [6].

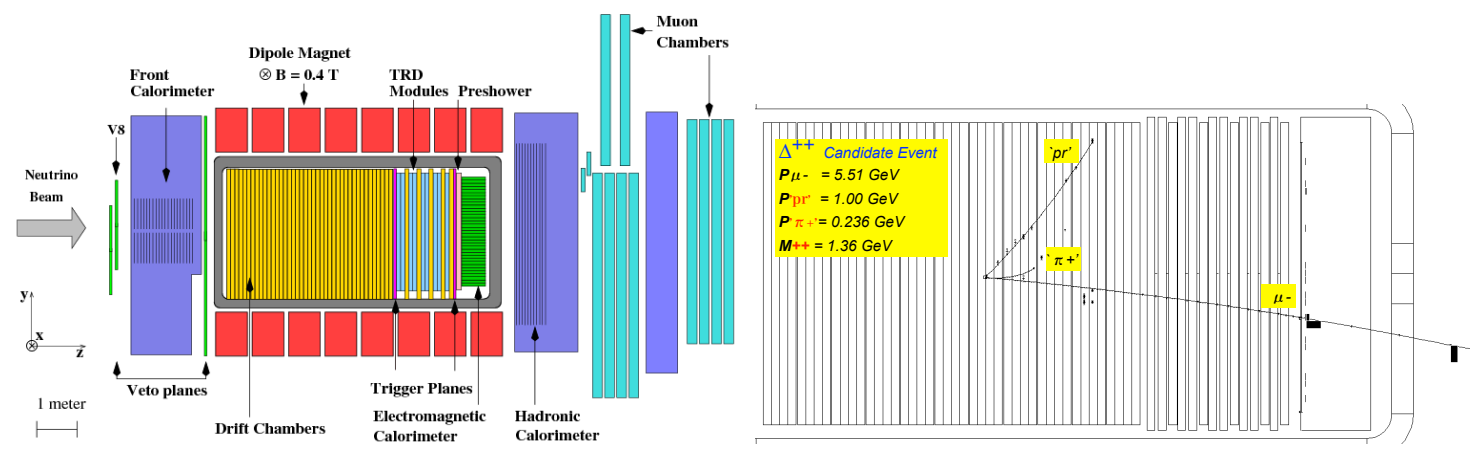

Figure 1: Diagram showing the components of the NOMAD detector (left) and a resonance candidate event in the NOMAD detector with a muon track and two positive hadron tracks.

Signal in this analysis is defined as charged current $v_{\mu}$ interactions $\left(v_{\mu}\right.$-CC) in the resonance region $(W<2.0 \mathrm{GeV})$ with at least two final-state positive hadrons (protons or $\pi^{+}$) above the detector threshold $\left(p_{p}>300 \mathrm{MeV}, p_{\pi^{+}}>80 \mathrm{MeV}\right)$. To select a clean sample of signal events, the events accepted into the analysis are required to have one negative track in the drift chambers identified as a muon by matching with track segments in both muon chambers, and two positive hadronic tracks reconstructed in the drift chambers (3-track topology, Fig.1 right). Neutral energy in the tracker or calorimeters is vetoed to reduce background events from the higher- $W$ region. Momentums of charged particles are measured by reconstructed track curvatures. Fig. 2 shows 
the invariant hadronic mass $W$ reconstructed from the measured final-state particle momentums in the analysis sample with both signal and background normalized to data. The selected sample is dominated by $W<2.0 \mathrm{GeV}$ signal events, with backgrounds contribution mostly from $W>$ $2.0 \mathrm{GeV}$ events, and some small contribution from $W<2.0 \mathrm{GeV}$ events without the required abovethreshold proton or $\pi^{+}$. The simulated background events are normalized to the sideband data defined as reconstructed $W>2 \mathrm{GeV}$, and subtracted from data in the signal region (reconstructed $W<2.0 \mathrm{GeV}$ ) to get a measurement of the raw signal.

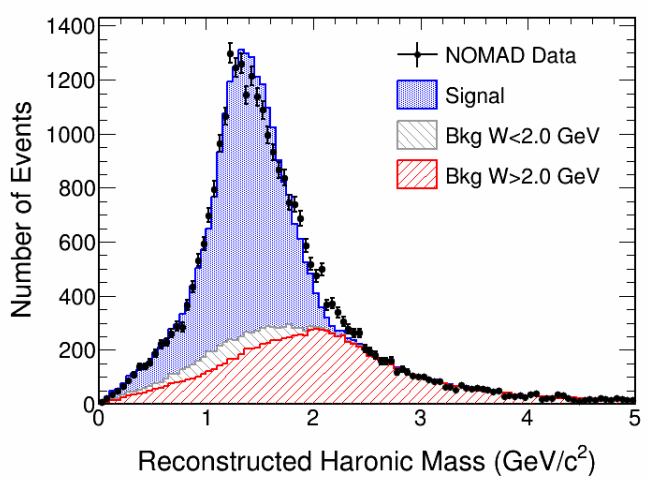

Figure 2: Reconstructed hadronic invariant mass of the selected 3-track sample. The simulated background events are normalized to the sideband data (reconstructed $W>2.0 \mathrm{GeV}$ ), and the signal events are normalized to the data in the signal region (reconstructed $W<2.0 \mathrm{GeV}$ ) with normalized background subtracted.
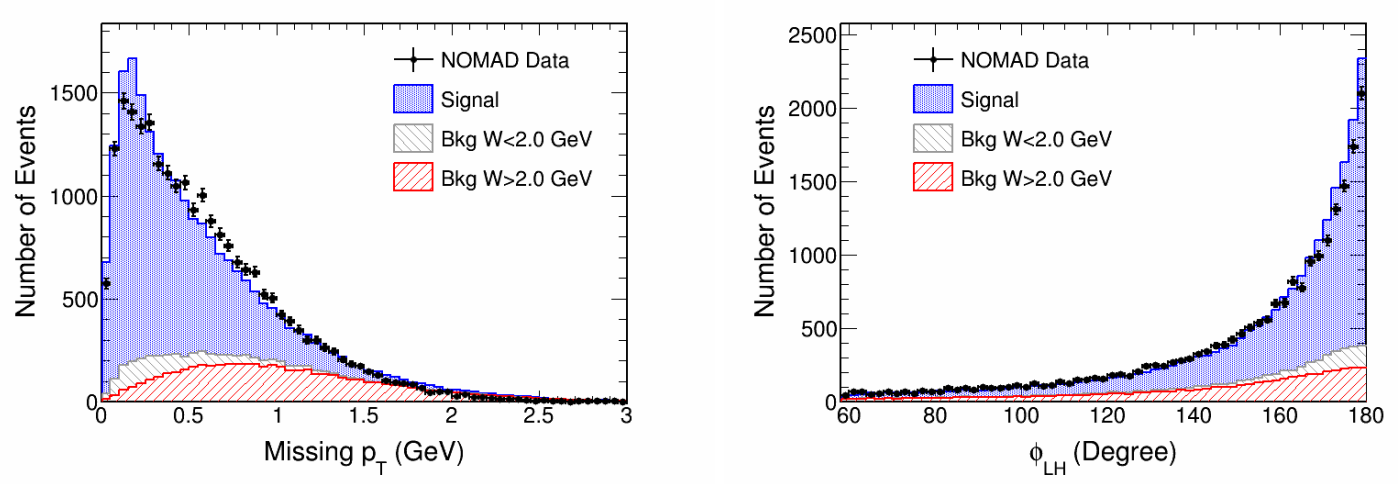

Figure 3: Comparison between data and simulation in transverse variables: missing $p_{T}$ (left) and $\phi_{L H}$ (right).

Fig. 3 compares data in the selected $W<2 \mathrm{GeV}$ signal region with simulation as functions of transverse kinematic variables that are sensitive to nuclear effects. The kinematic variables shown are the momentum imbalance on the plane transverse to the incoming neutrino beam direction (missing $p_{T}$ ), and the azimuthal angle between the muon momentum and hadronic momentum on 
the transverse plane $\left(\phi_{L H}\right)$. Discrepancies between data and simulation can be observed in both variables.

The background-subtracted raw data is corrected by detector acceptance and selection efficiency, and divided by the neutrino flux and number of target nucleons to get the total cross section as a function of neutrino energy (Fig. 4). Lower total cross section is observed compared to the GENIE prediction. Only statistical uncertainty is shown.

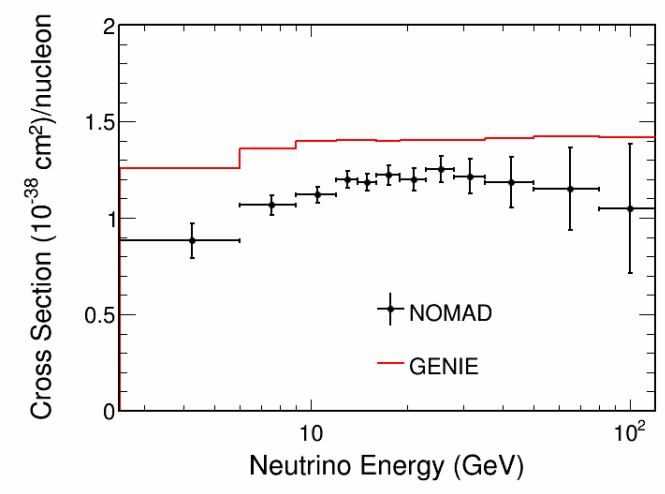

Figure 4: Measured cross section of $v_{\mu}$-CC interactions in the resonance region compared to the GENIE prediction. The error bars show statistical uncertainty only.

In summary, a preliminary study of $v_{\mu}$-CC events in the resonance region $(W<2.0 \mathrm{GeV})$ is performed. The total cross section as a function of neutrino energy is reported with statistical uncertainty only. The future goal of this work is to complete the analysis with systematic uncertainties, and report differential cross sections as functions of muon, proton and pion kinematics, as well as transverse variables to quantitatively constrain nuclear effects.

\section{References}

[1] D. Rein and L. M. Sehgal, Annals Phys. 133, 79 (1981).

[2] C. Andreopoulos et al., Nucl. Instrum. Meth. A 614, 87 (2010); C. Andreopoulos et al., arXiv:1510.05494v1 [hep-ph] (2015).

[3] F. Vannucci, Adv. High Energy Phys. 2014, 129694 (2014). doi:10.1155/2014/129694

[4] G. Acquistapace et al., CERN-ECP/95-14

[5] P. Astier et al., NIM A 515, 800-828 (2003)

[6] M. Gluck, E. Reya, A. Vogt, Z. Phys. C 53127 (1992) 